\title{
Lipases and the stress of obesity
}

\author{
Abbreviations: LPL, Lipoprotein lipase; HL, hepatic lipase; \\ HSL, hormone sensitive lipase
}

\section{Opinion}

Stress can be defined as an organism's response, which developed through phylogeny, in response to danger. ${ }^{1}$ The individual response is activated when the stimulus exceeds the subjective capacity of homeostatic mechanisms to confront the disruption of the internal environment. Every phenomenon has a physical component, determined by the nature or origin of the stress phenomenon, and another psychological component, which refers to how the body perceives this stimulus. The primary response to stress consists of the activation of the hypothalamic-pituitary-adrenal axis. ${ }^{2}$ Glucocorticoids stimulate the synthesis and release of catecholamines, especially in the adrenal medulla. Both circulating glucocorticoids and catecholamines reach the target tissues and prepare to confront the danger. The main metabolic abnormalities were hyperglycemia, increased lipolysis, increased heart rate and blood pressure, etc. Although it is known that stress is a risk factor in the development of atherosclerosis, little is known about its effect on lipid metabolism and lipoprotein lipase (LPL) and hepatic lipase (HL) activities.

The existing literature regarding the effect of stress on lipid metabolism. ${ }^{3,4}$ is sparse and, in the case of LPL, contradictory. ${ }^{5-7}$ It has been described in rats, in vivo and in vitro that adrenaline causes a decrease in the LPL activity in the vascular bed of adipocytes, but expression of the gene encoding the enzyme was not affected, ${ }^{8}$ in parallel with an increase in plasma LPL activity as well as the appearance of LPL activity in the liver, ${ }^{9,10}$ but there is still controversy about the regulatory mechanism. To further the knowledge of LPL in vivo regulation by adrenaline, a model of restraint stress in rats was used and applied either as chronic and acute conditions. ${ }^{11}$ Both chronic and acute stresses were reported to produce significant alterations in lipid and lipoprotein metabolism, ${ }^{11}$ and these changes were accompanied by a decrease in LPL activity in white adipose tissue and an increase in plasma. ${ }^{8}$ The possible role of blood flow in the regulation of LPL by catecholamines has also been studied. Because of its intravascular location, it is possible that LPL is sensitive to variations in the flow of blood through the capillaries. Thus, an increased flow could lead to an increase in the release of LPL from its anchor, which would lead to a decline in activity in the tissue without altering its synthesis. This would provide a mechanism for short-term regulation if the LPL (much faster than the regulation of enzyme synthesis), which has not been studied yet. It has been reported that acute immobilization stress increased blood flow in white adipose tissue (which reduces LPL activity) but not in muscle (in which LPL was unchanged). ${ }^{12}$ It has also been reported that LPL shows a circadian rhythm in adult rats ${ }^{13}$ that can be modified by soft stress. ${ }^{14}$ However, there are very few data linking stress caused by with lipolytic activity. In experimental animals, HL activity and mRNA were shown to decrease in the liver after hepatectomy, whereas activity was not detected in the plasma. However, adrenal HL activity did not vary post-surgery. ${ }^{15}$ This is very interesting because the steroidogenic organs, such as the adrenal gland, cannot synthesize the enzyme, which originates in the liver; however, given the need to capture cholesterol for the synthesis of catecholamines and glucocorticoids, such as in a stressful situation,
Volume 5 Issue 3 - 2017

\author{
Eva Pardina,' Júlia Carmona,' Joana \\ Rossell,' David Ricart-Jane,' Juan A Baena \\ Fustegueras, ${ }^{2}$ Julia Peinado-Onsurbe' \\ 'Department of Biochemistry and Molecular Biomedicine, \\ University of Barcelona, Spain \\ ${ }^{2}$ Surgery Unit, Hospital Universitari Arnau de Vilanova, Lleida, \\ Spain
}

Correspondence: Julia Peinado-Onsurbe, Department of Biochemistry and Molecular Biomedicine, Faculty of Biology, University of Barcelona, Diagonal 643, 08028 Barcelona, Spain, Tel (34)-(93)-402। 524/48, Fax (34)-(93)-402I559, Email jpeinado@ub.edu

Received: October 27, 2017 | Published: November 17, 2017

steroidogenic organs retain high levels of HL. It has also been observed that surgical stress leads to the recovery of the LPL activity and mRNA in the liver, and there are also changes in the expression of LPL and other proteins, such as albumin, actin, etc, in peripheral tissues. $^{16}$

Today, we tend to view the psychopathology of obesity as a consequence more than as a cause of obesity. Despite this positive point of view, the obese continue to suffer prejudice and discrimination in everyday life. Adverse psychosocial effects, together with the high incidence of cardiovascular disease and diabetes with morbid obesity, call dramatically for obesity treatment. ${ }^{17}$ The psychopathological profiles of the morbidly obese before surgery show depression, feelings of insecurity and despair, somatization, denial of emotional stress, difficulties in interpersonal relationships, poor social adjustment and low self esteem. After surgery, improvement is determined by the psychological effect of weight loss and even the occasional surgical complications, yet, despite the complications, these patients have less mental and emotional suffering. Apparently, these patients prefer a non-obese state with surgical complications to the obese state. ${ }^{17}$ Recently it has been suggested that adverse psychological states associated with chronic stress may lead to visceral obesity. ${ }^{18}$ A great portion of studies done on lipoproteins and/or lipolytic activity (LPL, HL, HSL, hormone sensitive lipase) and obesity have been conducted in obese or morbidly obese patients undergoing different kinds of surgery (mostly cholecystectomy) who, additionally, have participated in adipose tissue biopsies. ${ }^{19}$ But studies on the obese or morbidly obese undergoing bariatric surgery in which the stress of surgery has been added to a sharp weight loss, which can lead to significant changes in metabolism, are scarce. The extracellular localization of LPL clearly affects the mechanisms through which it could be regulated, such as allostery, phospho-dephosphorylation, etc. Several authors have concluded that LPL is regulated in the short term primarily through changes in its synthesis and intracellular processing. ${ }^{20}$ This seems particularly true in the changes that nutritional status of the individuous causes in the LPL activity of different tissues. ${ }^{21}$ However, changes that occur in other situations, such as stress, may use other control mechanisms that have not yet been described. 
Catecholamines can be seen as negative regulators of LPL in WAT for two reasons: first, adrenergic stimulation by catecholamines of the pancreas decreases insulin secretion, which potentially eliminates or reduces LPL in WAT; second, catecholamines and other modulators that increase cAMP levels decrease LPL activity by accelerating the inactivation or degradation of LPL inside adipocytes. ${ }^{22}$ Thus, people who have both an LPL and/or HL decrease and obesity have an increased risk for cardiovascular diseases. ${ }^{23}$ In population studies, adrenal hormones show strong statistical associations with centralization of body fat as well as obesity. There is considerable evidence from clinical, cellular and molecular studies that elevated cortisol, particularly when combined with secondary inhibition of sex steroids and secreted growth hormones, causes accumulation of fat in visceral adipose tissues as well as metabolic abnormalities (known as metabolic syndrome).

Hypertension is probably due to a parallel activation of the central sympathetic nervous system. Depression and 'small baby syndrome' as well as stress exposure in men and non-human primates are followed with time by similar central and peripheral abnormalities. Glucocorticoid exposure is also followed by increased food intake and 'leptin resistant' obesity, perhaps by disrupting the balance between leptin and neuropeptide $Y$ to the advantage of the latter. The consequence might be 'stress eating', which is a poorly defined entity. Factors that activate the stress in humans include psychosocial and socioeconomic handicaps, depressive and anxiety traits, alcohol and smoking, with some differences in profiles between personalities and genders.

\section{Acknowledgements}

This work was supported by the Ministry of Health and Consumer Affairs, Carlos III Health Institute (ISCIII) (PI11/01159 and PI15/00190 to JP-O PI15/00332 to JAB-F) and the FEDER Funds of the EU (European Fund for Regional Development: "A way of making Europe").English grammar and language have been corrected by American Journal Experts (www.journalexperts.com).

\section{Conflicts of interest}

The author declares there are no conflicts of interest.

\section{References}

1. Vigas N. Contribution to the understanding of the stress concept en: Catecholamines and stress: recent advances. In: E Ursding, et al. editors. Netherlands: North Holland Publishing Co; 1980. p. 572-578.

2. Martí O, Wardrobe A. Neuroendocrinological modifications to stress. In: Francesc Artigas editor. Neuroscience of the Catalan Society of Biology. 1996. p. 171-185

3. Hershock D, Vogel HV. The effects of immobilization stress on serum triglycerides, nonesterified fatty acids, and total cholesterol in male rats after dietary modifications. Life Sci. 1989;45(2):157-165.

4. Armario A, Montero JL, Balasch J. Sensitivity of corticosterone and some metabolism variables to graded levels of low intensity stresses in adult male rats. Physiol Behav. 1986;37(4):559-561.

5. Borbola J, Gecse A, Karady S. The activity of lipoprotein lipase in rat heart after tourniquet stress. Adv Exp Med Biol. 1972;33(0):387-393.

6. Ashby P, Robinson DS. Effects of insulin glucocorticoids and adrenaline on the activity of rat adipose-tissue lipoprotein lipase. Biochem $\mathrm{J}$. 1980;188(1):185-192.
7. Hülsman W, Dubelaar ML. Lipoprotein lipase and stress hormones: studies with glucocorticoids and cholera toxin. Biochim Biophys Acta. 1986;875(1):69-75.

8. Ballart X, Siches M, López Tejer D, et al. Lipoprotein lipase activity is increased in medium of adipocytes incubated with isoproterenol: a possible link with its increase in plasma of rats treated with adrenaline or beta-adrenergic agonist. Biochimie. 2003;85:971-982.

9. Ball KL, Speake BK, Robinson DS. Effects of adrenaline on the turnover of lipoprotein lipase in rat adipose tissue. Biochim Biophys Acta. 1986;877(3):399-405.

10. Knobler H, Chajek Shaul T, Stein O, et al. Modulation of lipoprotein lipase in the intact rat by cholera toxin-an irreversible agonist of cyclic AMP. Biochim Biophys Acta. 1984;795(2):363-371.

11. Ricart Jané D, Rodríguez Sureda V, Benavides A, et al. Immobilization stress alters intermediate Comorbidities Associated with Morbid Obesity 31 metabolism and circulating lipoproteins in the rat. Metabolism. 2002;51:925-931.

12. Ricart Jané D, Cejudo Martín P, Peinado Onsurbe J, et al. Immobilization stress alters intermediate metabolism and circulating lipoproteins in the rat. Metabolism. 2002;51(7):925-931.

13. Benavides A, Siches M, Llobera M. Circadian rhythms of lipoprotein lipase and hepatic lipase activities in the intermediate metabolism of the adult rat. Am J Physiol. 1998;275(3):811-817.

14. Abellán R, Benavides A, Díez Noguera A, et al. Effects of mild stress by isolation on metabolic circadian rhythms in the adult rat. Am J Physiol. 2002;22:417-422.

15. Sabugal R, Robert MQ, Julve J, et al. Decrease in the expression of hepatic lipase activity following partial hepatectomy. Biochim Biophys Acta. 1996;1302:193-198.

16. Palmero E, Ricart D, Llobera M, et al. Partial hepatectomy and/or surgical stress provoke changes in the expression of lipoprotein lipase and actin in liver and extrahepatic tissues. Biochim Biophys Acta. 1999;1441(1):6168.

17. Van Gemert WG, Severeijns RM, Greve JW, et al. Psychological functioning of morbidly obese patients after surgical treatment. Int J Obes Relat Metab Disord. 1998;22(5):393-398.

18. Montague CT, Prins JB, Sanders L, et al. Depotrelated gene expression in human subcutaneous and omental adipocytes. Diabetes. 1998;47(9):13841391.

19. Ham JM, Slack WW. Lipoprotein lipase activity in patients before and after minor surgical operations. Clin Chim Acta. 1969;25:417-422.

20. Doolittle MH, Ben Zeev O, Davis RC, et al. Lipases and obesity: posttranslational regulation of lipoprotein lipase, Obesity towards a molecular approach. In: GA Bray, et al. editors. New York, USA: Wiley-Liss; 1990. p. $237-246$.

21. Doolittle MH, Ben Zeev O, Elovson J, et al. The response of lipoprotein lipase to feeding and fasting. Evidence for posttranslational regulation. $J$ Biol Chem. 1990;265(8):4570-4577.

22. Bagby GJ, Pekal PH. Lipoprotein lipase in trauma and sepsis. In Lipoprotein lipase. In: Borensztajn editor. Chicago: Evener Publishers Inc; 1987 . p. 247-274.

23. James RW, Brulhart Meynet MC, Lehmann T, et al. Lipoprotein distribution and composition in obesity: their association with central adiposity. Int J Obes Relat Metab Disord. 1997;21:1115-1120. 